\title{
Impact of Toxin-Specific Antibodies on the Adjuvanticity and Inflammatory Effects Induced by Parenterally Administered Escherichia coli heat-Labile Toxin
}

\author{
Mariana de Jesus Cintra ${ }^{1}$, Denicar Lina Nascimento Fabris Maeda ${ }^{1,2}$, Camila Mathias-Santos ${ }^{1}$, \\ Lennon Ramos Pereira ${ }^{1}$, Wilson Barros Luiz ${ }^{1}$, Luís Carlos de Souza Ferreira ${ }^{{ }^{*}}$ and Juliana \\ Falcão Rodrigues ${ }^{1,2}$ \\ ${ }^{1}$ Vaccine Development Laboratory, Department of Microbiology, Biomedical Sciences Institute, University of São Paulo, São \\ Paulo, Brazil. \\ ${ }^{2}$ Department of Parasitology, Biomedical Sciences Institute, University of São Paulo, São Paulo.
}

Received: 19 April, 2018; Accepted: 24 May, 2018; Published: 29 May, 2018

*Corresponding authors: Dr. Luis C.S. Ferreira, Dra. Juliana F. Rodrigues, Vaccine Development Laboratory, Department of Microbiology, Biomedical Sciences Institute, University of São Paulo, São Paulo, Brazil, E-mail: lcsf@usp.br, jufalcao@usp.br

\begin{abstract}
Introduction: Heat-labiletoxins (LT), produced by enterotoxigenic Escherichia coli (ETEC) strains, exert potent adjuvant effects when admixed or linked to antigens delivered via mucosal, transcutaneous or parenteral routes. There is limited information regarding the impact of preexisting immunity on the immunomodulatory properties of LT, which is frequently observed among people infected with ETEC.
\end{abstract}

Aims: In the present study, we evaluated the effect of anti-LT antibodies on the adjuvant and inflammatory activities triggered by LT admixed with a specific vaccine antigen following subcutaneous administration to mice.

Material and Methods/Results: Animals were immunized with dengue virus nonstructural protein (NS1), as model antigen, in combination with native LT in the presence of LT-specific antibodies. Exposure to anti-LT antibodies did not impair the humoral adjuvanticity of LT regarding to the magnitude of the serum anti-NS1 IgG titers. In addition, anti-toxin antibodies did not reduce neutrophil migration nor edema formation after s.c. administration of LT. Nonetheless, administration of LT admixed with anti-LT antibodies changed the local cytokine production profile and modulated the NS1specific T cell responses to a Th1-type pattern.

Conclusion: These results indicate that preexisting immunity does not affect the humoral adjuvant activities but may modulate different aspects of both innate and adaptive immune responses induced by parenterally administered LT.

Keywords: Heat-Labile Toxin; Vaccine Adjuvant; Preexisting Immunity; Inflammatory Response; Adaptive Immunity; Vaccine;

\section{Introduction}

ETEC strains are considered important etiologic agents of the childhood diarrhea and represent the most common cause of the traveler's diarrhea in endemic areas. The pathogenesis induced by ETEC is associated with the production of both colonization factors, which mediate adhesion to gut cells, and enterotoxins, particularly heat-stable and/or heat-labile toxin families, involved specifically with cytotoxic effects. LT toxins are characterized by an enzymatically active A subunit noncovalently linked to a pentameric ring of B polypeptides, which is responsible for binding to cell surface receptors such as GM1 ganglioside [1,2]. Together with cholera toxin (CT), LT shows also remarkable adjuvant activities following mucosal and parenteral administration improving both humoral and cellular immune responses against target antigens[3-6]. However, the inherent toxicity of LT, even after inoculation of low amounts, has precluded its use as a vaccine adjuvant for humans, particularly under mucosal route $[3,4,7,8]$. On the other hand, detoxified LT mutants and natural variants have demonstrated safety features after parenteral inoculation[8-11].Thus, parenteral administration of LT-derivatives represents a new and promising alternative for clinical use of this class of vaccine adjuvant.

Preexisting immunity may compromise the performance of vaccines, particularly those based on microorganisms as antigendelivery systems, such as adenovirus and Salmonella [12-14]. Such restriction may also apply to protein adjuvant, such as derivatives of LT and CT. Individuals naturally or deliberately challenged with ETEC or Vibrio cholera strains mount local and systemic antibody responses to LT[15-18]. Some reports demonstrate that the rate of seroconversion directed towards LT is high, ranging from $75 \%$ to $92 \%$ at the first infection and reaching about $50-60 \%$ under rechallenge[15,18,19]. Additionally, it has been seen that the antiLT antibodies in serum samples or in lymphocyte supernatants remained at increased levels following rechallenge with wild-type ETEC strain or an inactivated oral Vibrio cholera vaccine[18,20]. Collectively, these findings suggest that the natural exposure to LT or CT in endemic countries results in individuals with bloodcirculating LT-specific antibodies, which could access mucosal 
compartments and particularly systemic tissues, and might hamper the adjuvant properties of parenterally administered LT derivatives incorporated into vaccine formulations.

Previous studies have demonstrated that preexisting immunity to CT does not decrease induction of the antigenspecific antibody responses following mucosal immunization with attenuated forms of this toxin [21,22]. Ugozzoli and coworkers (2001) showed that previous immunity to LT did not impact the humoral adjuvant activity following administration via mucosal routes [23]. Considering that no information regarding the impact of preexisting immunity on the adjuvanticity of LT derivatives administered via parenteral routes exists, the present study was designed to evaluate the impact of toxin-specific antibodies on the innate and adaptive immune responses, including local inflammatory effects, associated with the use of LT as a parenteral adjuvant. For that purpose, we combined a recombinant form of the dengue type 2 virus non-structural protein 1 (NS1) with type 1 LT produced by wild type ETEC strains and immunized mice via the subcutaneous route. Our results demonstrated that preexisting immunity to LT did not affect the specific antibody responses to NS1. However, local proinflammatory cytokine environment induced by LT was changed in the presence of anti-LT antibodies resulting in increased levels of IL- 6 and IL-12 leading to more biased Th1 response profile.

\section{Material and Methods}

NS1 antigen and LT adjuvant. Recombinant NS1 protein was obtained as described by Amorim and coworkers (2010)[24]. E. coli BL21 strain harboring the plasmid pML19, which encoding holotoxin LT derived from reference ETEC H10407, was used for LT production[25]. Briefly, the recombinant E. coli was grown in Terrific Broth for $18 \mathrm{~h}$ at $37^{\circ} \mathrm{C}$ with vigorous agitation (200 r.p.m. in a rotary shaker). The bacterial cell sediment was mechanically disrupted using an homogenizer (Artpeças, São Paulo, Brazil) and clarified cell extracts were applied into the immobilized D-galactose resins (Pierce, Thermo Fisher Scientific, Waltham, Massachusetts, USA) for purification of LT AB5 holotoxin, which consists of an A subunit and a pentameric B subunit, as previously described [25]. Quantification of the purified proteins was carried out by spectrophotometry (Gene Quant spectrophotomer GE Amersham Biosciences, Buckinghamshire, UK) using the formula: concentration $(\mathrm{mg} / \mathrm{ml})=\left(\right.$ absorbance at $280 \mathrm{~nm} / \mathrm{n}^{\circ}$ tryptophan amino acid residues $x 5,690+n^{\circ}$ tyrosine amino acid residues $x$ $1,280) \times$ molecular mass (Da), as previously described (Edelhoch, 1967). The purified proteins were heated at $100{ }^{\circ} \mathrm{C}$, submitted to $17.5 \%$ SDS-PAGE and, then, evaluated by immunoblot with an anti-LT serum, previously obtained from mouse immunizations, or a monoclonal anti-NS1 antibody kindly provided by Dra. Roxane M. F. Piazza. The proteins were also submitted to an endotoxin removal step using $\varepsilon$-poly-L-lysine resins according to the manufacturer's instructions (Pierce $® H i g h-C a p a c i t y$ Endotoxin Removal Resin, Thermo Fisher Scientific, Waltham, Massachusetts, USA). After the treatment, the purified protein samples contained $<0.017 \mathrm{EU} / \mu \mathrm{g}$ of protein, as measured by Limulus amebocyte lysate method (Lysate LAL QCL-1000, Lonza, Basel, Switzerland).

\section{Treatment of LT with anti-toxin antibodies or soluble GM1}

The treatments encompassed the incubation of LT with anti-LT sera $(0.7 \mu \mathrm{g}$ of specific IgG/ $\mu$ g of toxin) or soluble GM1 ganglioside ( $0.5 \mu \mathrm{g}$ of GM1/ $\mu$ g of toxin) (Sigma-Aldrich, St. Louis, MO, USA) in a water bath incubator at $37^{\circ} \mathrm{C}$ for $1 \mathrm{~h}$, in a final volume of $20 \mu \mathrm{l}$, according to previous reported procedures[10,26]. The NS1 antigen $(10 \mu \mathrm{g})$ was incubated with anti-NS1 sera $(7 \mu \mathrm{g}$ of specific IgG) as described above and used as negative control for the evaluation of proinflammatory effects.

\section{LT cytotonic assay in Y1 cell culture}

The cytotonic assay was performed as previously reported[5]. The Y1 cells $\left(5 \times 10^{5}\right.$ cells $\left./ \mathrm{ml}\right)$ were exposure to LT $(10 \mu \mathrm{g} / \mathrm{ml})$ previously treated or not with the anti-LT sera or soluble GM1. After incubation for $8 \mathrm{~h}$, the cytotonic effects were assessed and images from representative fields of each treatment were captured using digital inverted microscope (EVOS FL Imaging System, Thermo Fisher Scientific).

\section{Mice and ethics statement}

Female BALB/c or C57BL/c mice (6-8 weeks old) used in the experiments were obtained from Isogenic Mouse Breeding Facility of Department of Parasitology, Institute of Biomedical Sciences - USP. The protocols involving animal handling were approved by the Ethics Commission on Animal Use (CEUA 006/03) of the Institute of Biomedical Sciences at the University of São Paulo (USP) in accordance with guidelines of the National Council of Animal Experimentation (CONCEA).

\section{Immunizations}

BALB/c mice were subcutaneously (s.c.) immunized with three doses of NS1 alone $(20 \mu \mathrm{g})$ or NS1 co-administered with LT $(1 \mu \mathrm{g})$ previously treated or not with anti-LT serum $(0.7 \mu \mathrm{g})$ or soluble purified GM1 $(0.5 \mu \mathrm{g})(\mathrm{n}=5 \mathrm{mice} /$ group$)$. Animals were bled two weeks after each dose by the submandibular vein. Serum samples were kept at $-20^{\circ} \mathrm{C}$. Mice were euthanized by $\mathrm{CO}_{2}$ asphyxiation two weeks after the last dose and spleen cells were harvested for cytokine analysis.

\section{Determination of NS1 or LT-specific IgG titers}

Measurements of serum anti-NS1 or anti-LT IgG titers were carried out as previously reported[24,27]. Briefly, microtiter plates (Nunc Maxisorp-Thermo Fisher Scientific) were coated with LT $(1 \mu \mathrm{g} / \mathrm{ml})$ or NS1 $(1.5 \mu \mathrm{g} / \mathrm{ml})$ for $16-18 \mathrm{~h}$ at $4^{\circ} \mathrm{C}$. Two-fold serial dilutions of the tested sera were added to the plates, washed and incubated with horseradish peroxidase-conjugated goat antimouse IgG antibodies (Sigma-Aldrich, St. Louis, MO, USA) diluted to 1:3,000.The ELISA reactions were read at $492 \mathrm{~nm}$ in EpochTM Multi-Volume Spectrophotometer (BioTek Instruments, Vermont, USA). The results are expressed as titers, defined as the highest sample dilution with an $\mathrm{A}_{492 \mathrm{~nm}} \geq 0.2$ above pre-immune sera.

\section{Determination of the secreted cytokine profiles of spleen cells}

The pools of spleen cells $\left(1 \times 10^{7}\right.$ cells $/ \mathrm{ml}$, in 12 -well plates $)$ 
collected from each immunization group were cultured with NS1 $(10 \mu \mathrm{g} / \mathrm{ml})$ for $48 \mathrm{~h}$ at $37^{\circ} \mathrm{C}$ and $5 \% \mathrm{CO}_{2}$ in RPMI medium supplemented with $10 \%$ FBS, $2 \mathrm{mM}$ L-glutamine, $1 \mathrm{mM}$ sodium pyruvate, $0.5 \mathrm{mM}$ essential amino acids, $1 \mathrm{mM}$ nonessential amino acids, $10 \mathrm{mM}$ HEPES buffer and $50 \mathrm{U} / \mathrm{ml}$ of penicillinstreptomycin(Gibco, Thermo Fisher Scientific). Cell culture supernatants were assayed for cytokines by ELISA (BD OptEIA ${ }^{\mathrm{TM}}$ - Mouse IL5 ELISA Set and BD ${ }^{\mathrm{TM}}$ Cytometric Bead Array (CBA) Mouse Th1/Th2/Th17 cytokine kit; BD Biosciences, San Jose, CA, USA) according to the manufacturer's instructions.

\section{Edema formation and secretion of myeloperoxidase (MP0) at subcutaneous tissues}

The assays were based on protocols previously reported $[8,28]$ The local edema sizes were measured at different times according to the formula: M1 x M2, where M1 and M2 represent transverse and longitudinal measurements of the swelling region, respectively. For MPO measurements, skin biopsies (100 $\mathrm{mg}$ ) were dispersed twice for $45 \mathrm{sec}$ each (T10 Basic IKA UltraTurrax Disperser - Sigma-Aldrich) in sodium phosphate buffer (0.02 $\mathrm{M} \mathrm{NaH}_{2} \mathrm{PO}_{4}, 0.015 \mathrm{M} \mathrm{Na}_{2}$ EDTA, $0.1 \mathrm{M} \mathrm{NaCl}, \mathrm{pH} 4.7$ ) in a final volume of $2 \mathrm{ml}$. After centrifugation, the sediments were treated with $0.05 \mathrm{M}$ sodium phosphate buffer (pH 5.4) containing $0.5 \%$ hexadecyltrimethylammonium bromide, freeze-thawed (3x), and, then, centrifuged again. The supernatants $(50 \mu \mathrm{l})$ were incubated for $5 \mathrm{~min}$ at $37^{\circ} \mathrm{C}$ with BD OptEIA TMB substrate reagent $(150 \mu \mathrm{l})$ in triplicate at 96-well microtiter plates (Corning Costar - Sigma Aldrich) and the absorbance was read at $450 \mathrm{~nm}$ (Epoch, BioTek Instruments). MPO analyses were expressed as $\mathrm{A}_{450 \mathrm{~nm}} / \mathrm{mg}$ of total protein measured by bicinchoninic acid method (BCA Protein Assay, Pierce - Thermo Fisher Scientific).

\section{Measurement of proinflammatory cytokines}

Collection of skin interstitial fluids was carried out by centrifugation according to a previously reported protocol[29]. Skin biopsies $\left(1.5 \mathrm{~cm}^{2}\right)$ were harvested, placed with the edematous tissue facing down on a $40 \mu \mathrm{M}$ nylon cell strainer(BD Biosciences) coupled to polypropylene conical tubes $(50 \mathrm{ml})$ and centrifuged $\left(300 \mathrm{xg}, 30 \mathrm{~min}, 4^{\circ} \mathrm{C}\right)$. The samples were re-centrifuged for 10 min to pellet the contaminating cells and the supernatants were collected for proinflammatory cytokine quantification by ELISA (BD OptEIA ${ }^{\mathrm{TM}}$ - Mouse ELISA Sets, BD Biosciences) according to the manufacturer's instructions.

\section{Statistical analyses}

The results were analyzed with Graph Pad Prism 5 software (La Jolla, CA, USA) using two-way analysis of variance (ANOVA) and Bonferroni's post-hoc test for comparisons of means. The $p$ values $<0.05$ were considered statistically significant.

\section{Results}

\section{LT purification and treatment with neutralizing agents of the cytotoxic effects}

Initially, recombinant forms of the antigen (dengue virus NS1 protein) and the adjuvant (type 1 LT from reference ETEC H10407 strain) were purified by affinity chromatography at high purity levels (Figure 1). The purified LT was used in the immunological assays associated or not to neutralizing agents. The amounts of antibodies or GM1 receptor required to neutralize the LT present in the vaccine formulations were determined in Y1 cells. Incubation of LT with anti-LT antibodies $(0.7 \mu \mathrm{g}$ of antibodies $/ \mu \mathrm{g}$ of LT) or with soluble GM1 (0.5 $\mu \mathrm{g}$ of GM1/ $\mu \mathrm{g}$ of LT) neutralized the in vitro toxicity of LT to Y1 cells (Figure 2).

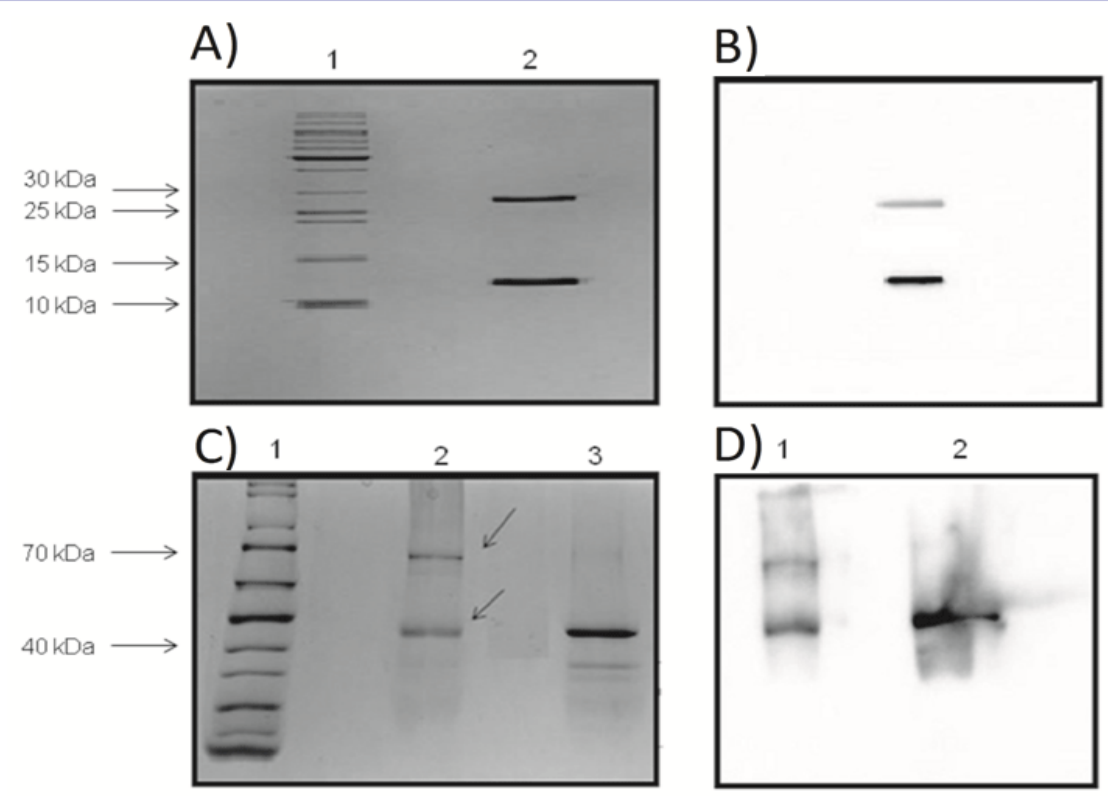

Figure 1: Characterization of the LT adjuvant and the NS1 antigen. Coomassie blue staining SDS-PAGE loaded with LT (A) or NS1 (C). Immunoblot developed with anti-LT serum (B) or monoclonal anti-NS1 antibody (D) on the purified proteins. (A and B) Lane 1, Molecular weight standards (kD); lane 2, boiled LT holotoxin, showing dissociated A and B subunits (28 kDa and $11.5 \mathrm{kDa}$, respectively). (C and D) Lane 1, Molecular weight standards (kD); lane 2, non-boiled NS1 protein; lane 3, boiled NS1 protein. (C) The arrows indicate dimers and monomers (43kDa) of NS1 protein. 

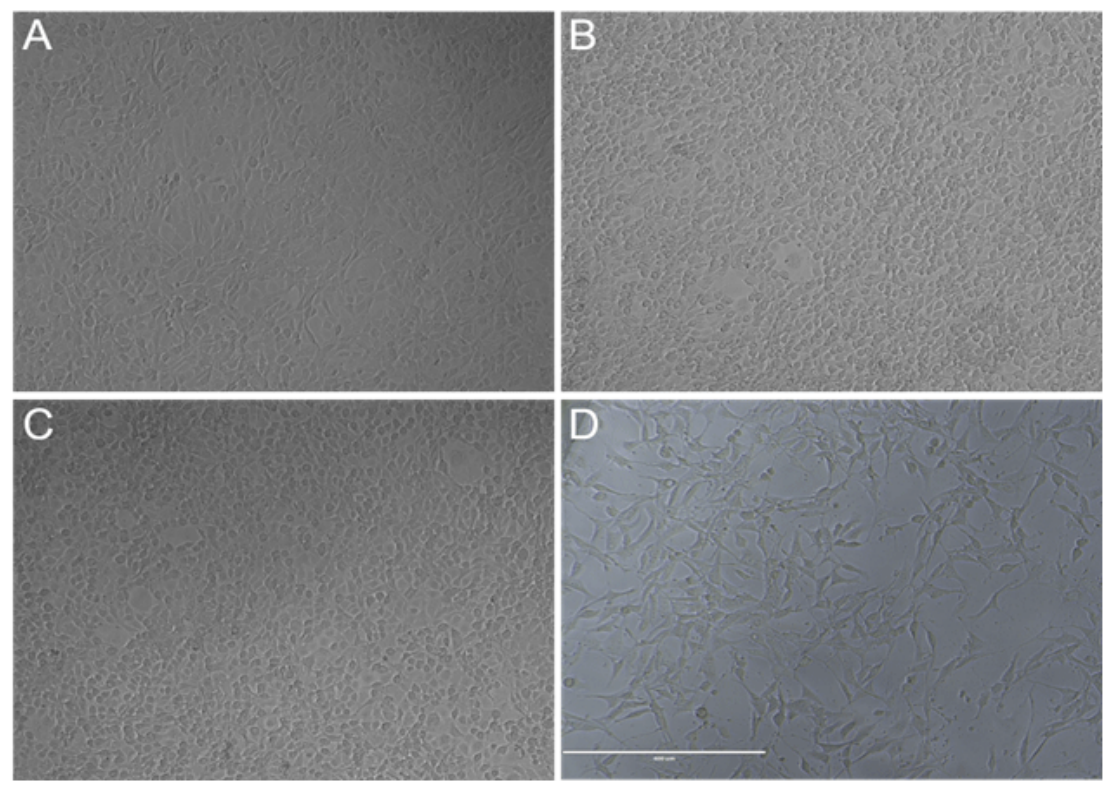

Figure 2: In vitro neutralization of LT cytotonic effect by anti-LT antibodies or soluble GM1 receptor.

The in vitro cytotonic assay evaluates the LT cytotonic effect on Y1 eukaryotic cells and the neutralizing ability of the anti-LT antibodies (700 ng of anti-LT IgG), present in sera harvested from mice immunized with LT, or soluble ganglioside GM1 receptor (500 ng) previously incubated with purified LT $(1 \mu \mathrm{g})$. The Y1 cells $\left(5 \times 10^{5} / \mathrm{ml}\right)$ were seeded in plates and incubated with $100 \mu \mathrm{L}$ of the LT mixed or not with each neutralizing agent. A, Y1 cells not treated (negative control); B, Y1 cells treated with LT (positive control); C and D, Y1 cells treated with LT+GM1 (C) or LT+Ab (D). Data are representative of two independent experiments.

\section{Impact of toxin-specific antibodies on the inflammatory responses induced by $\mathrm{LT}$}

Intradermal (i.d.) administration of LT to C57BL/6 mice induces strong local inflammatory reactions (neutrophil infiltration and edema) that may persist for several days [8]. The impact of toxin-specific antibodies on the LT-associated inflammatory reactions was evaluated in mice s.c. inoculated with different amounts of LT and, subsequently, monitored for edema formation and local production of MPO. As shown in Figure 3A and $\mathrm{B}$, edema formation peaked $48 \mathrm{~h}$ after inoculation of $10 \mu \mathrm{g}$ of LT and BALB/c mice controlled the inflammatory response more efficiently than C57BL/6 mice. Edema formation and MPO production were measured, both in BALB/c and C57BL/6 mice, after administration of $10 \mu \mathrm{g}$ of LT previously incubated with 7 $\mu \mathrm{g}$ of anti-LT antibodies (LT-Ab) or with $5 \mu \mathrm{g}$ of soluble GM1 (LTGM1) (Figure 3C to F). Inoculation of NS1 immune complexes (NS1 protein plus anti-NS1 serum) did not induce edema and neutrophil recruitment into s.c. tissue (data not shown). In addition, mice inoculated with LT treated with GM1 showed significantly smaller edema and lower local MPO production than animals inoculated with LT (Figure 3C to F). In contrast, mice inoculated with LT previously incubated with anti-LT antibodies developed edema sizes and local neutrophil infiltration similar to mice inoculated only with LT (Figure 3C to F). We also measured the local cytokine production in mice s.c. inoculated with LT admixed with anti-LT antibodies or purified GM1. The presence of IL-10, TNF- $\alpha$ and IFN- $\gamma$ was not detected in tissue samples of all tested groups. In contrast, inoculation of LT admixed with anti-LT antibodies enhanced the production of IL- 6 and IL-12, but did not interfere with the IL-1 $\beta$ levels induced by LT (Figure
4). Additionally, pre-exposure of LT to purified GM1 strongly suppressed the local production of IL-6, IL-12 and IL-1 $\beta$ secretion (Figure 4). These findings demonstrate that treatment with GM1 abrogates the local LT inflammatory effects; whereas anti-LT antibodies did not ablate the LT local inflammatory reactions but modulate the pro-inflammatory cytokines secreted by local cells.

\section{Exposure to antibodies modulates the adaptive immunity cytokine responses induced by LT}

The impact of the preexisting immunity was evaluated with LT-adjuvanted vaccine formulations in which LT was incubated with anti-LT antibodies before inoculation into BALB/c mice. Mice were immunized with vaccine formulations containing NS1 and LT previously admixed with anti-LT antibodies or GM1. As shown in Figure 5A, previous incubation of LT with anti-LT antibodies did not reduce the immunogenicity of the toxin, as measured by the amount of anti-LT antibodies in vaccinated mice. In contrast, mice immunized with LT previously admixed with GM1 showed significantly lower anti-LT responses (Figure 5A). These results indicated that previous exposure to LT-specific antibodies did not impact the immunogenicity of LT, while administration of LT associated to GM1 markedly reduced the immunogenicity of the toxin.

Regarding LT adjuvanticity, in vitro incubation of LT and anti-LT antibodies or GM1 did not impact the adjuvant effects of LT, as measured by the induced serum anti-NS1 IgG responses (Figure 5B). Similarly, the antigen-specific IgG subclass responses detected in mice immunized with LT and NS1 were not altered after co-administration of the vaccine formulation containing anti-LT antibodies or GM1. Concerning the T cell adjuvant effects, 

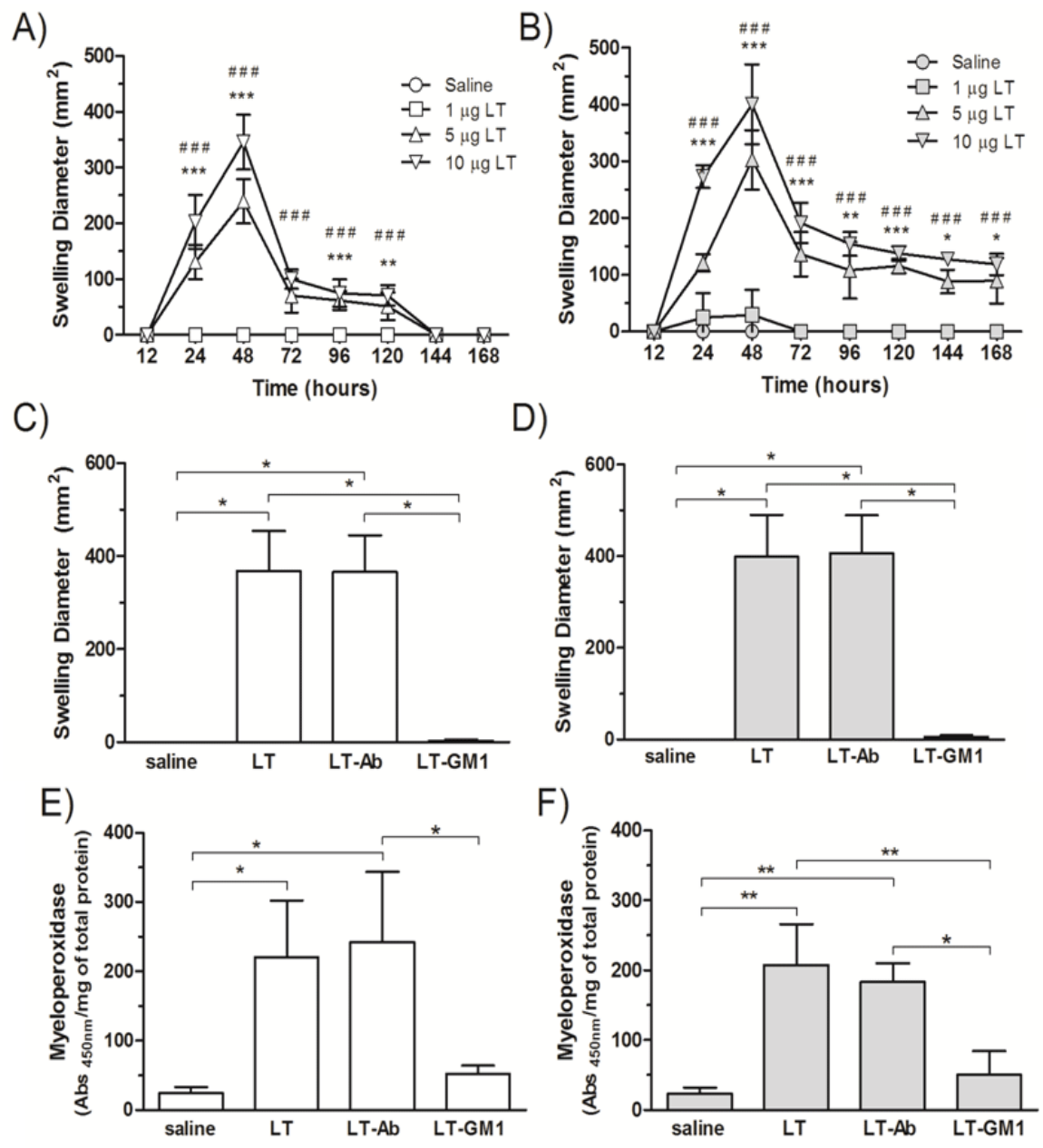

Figure 3: Inflammatory responses induced by LT-Ab immune complexes and LT admixed with GM1.

LT was administered to BALB/c (A, C and E; white symbols and bars) or C57BL/6 (B, D and F; grey symbols and bars) mice via the s.c. route. (A and B) Edema formation was measured up to 7 days after inoculation of different LT amounts. (C to F) Edema sizes (C and D) or MPO levels at the skin tissue ( $\mathrm{E}$ and F) measured $48 \mathrm{~h}$ after administration of LT (10 $\mu \mathrm{g} / \mathrm{mouse})$ alone or admixed with anti-LT sera or GM1. Data represent means \pm SE of three independent experiments. Statistical significant differences in comparison with negative control group (saline) or among groups indicated by brackets are shown: ${ }^{*} \mathrm{p}<0.05{ }^{* *} \mathrm{p}<0.01$; \#\#\# or ${ }^{* * *} \mathrm{p}<0.001$.
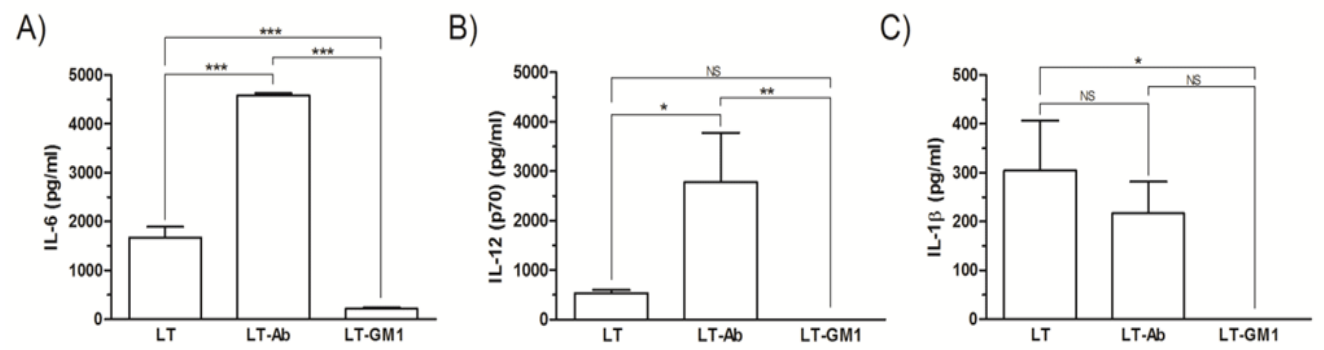

Figure 4: Pro-inflammatory cytokine profiles induced by LT treated with anti-LT antibodies or GM1.

Cytokines were measured at skin tissues harvested from BALB/c mice, $24 \mathrm{~h}$ after s.c. inoculation of LT admixed, or not, with anti-LT sera or GM1. Data represent means $\pm \mathrm{SE}$ of three independent experiments. Statistical significant differences are shown among groups indicated by the brackets: ${ }^{*} \mathrm{p}<$ $0.05 ;{ }^{* *} \mathrm{p}<0.01 ;^{* * *} \mathrm{p}<0.001$. NS: values without statistically significant differences.

Citation: Jesus Cintra MD, Nascimento Fabris Maeda DL, Luis C.S. Ferreira, et al. (2018) Impact of Toxin-Specific Antibodies on the Adjuvanticity and Inflammatory Effects Induced by Parenterally Administered Escherichia coli heat-Labile Toxin. Int J Vaccine Res 3(1): 1-9. 
A)

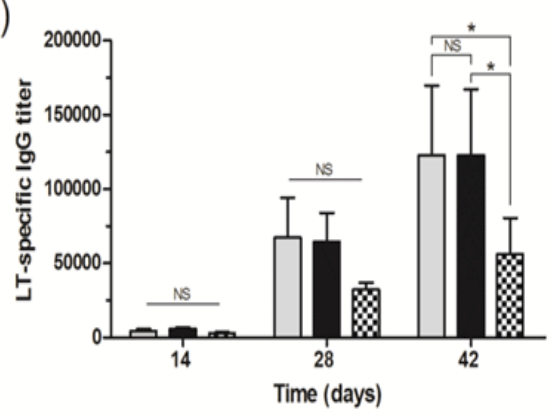

C)

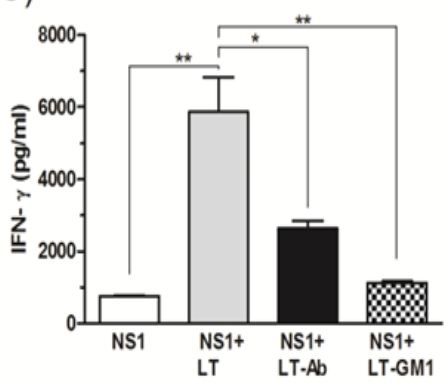

B)

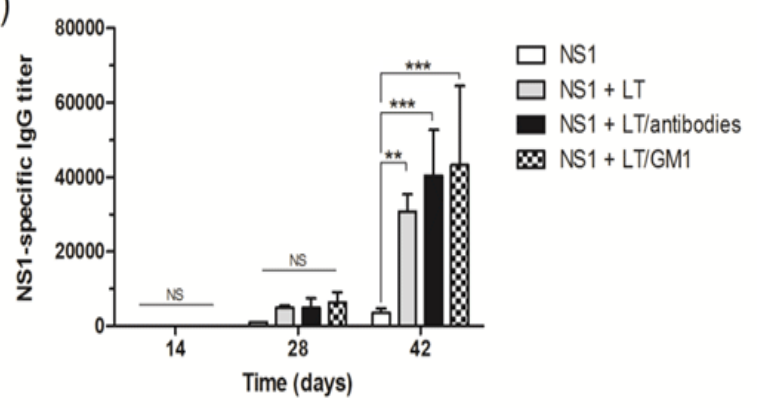

E)

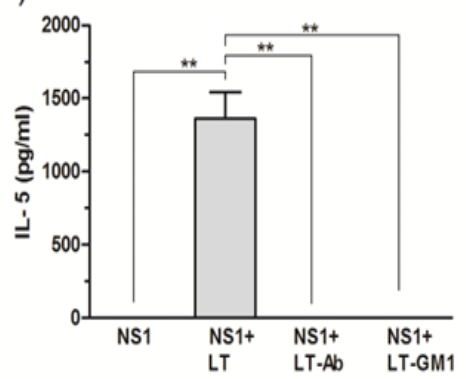

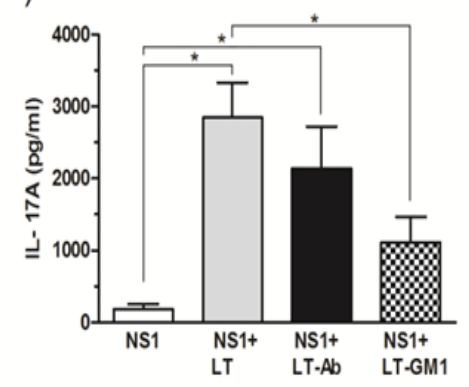

Figure 5: Evaluation of the immunogenicity and adjuvant effects induced by LT exposed to anti-LT antibodies or soluble GM1 receptor. BALB/c mice were s.c. immunized with three doses of NS1 antigen or NS1 admixed to LT, which was previously incubated or not with specific anti-LT or GM1. (A and B) Two weeks following the third dose, serum samples were harvested and anti-LT (A) or anti-NS1 (B) IgG titers were determined by ELISA. (C,D and E) Two weeks following the third dose, spleen cells were harvested and in vitro stimulated with the NS1 antigen. The cytokines present in cell culture supernatants were measured by ELISA. Data represent means \pm SE of three independent experiments. Statistical differences between the groups are indicated by the brackets: ${ }^{*}<<0.05$; ${ }^{* *} p<0.01 ;{ }^{* *} p<0.001$. NS: values without statistically significant differences.

BALB/c mice vaccinated with LT previously incubated with GM1 drastically reduced expression of IFN- $\gamma$, IL5 and IL-17A (Figure 5C to E). Incubation of LT with anti-LT antibodies did not affected production of IL-17A, but decreased the expression of IFN- $\gamma$ by $\sim 2$-fold and abrogated secretion of IL-5 (Figure 5C to E). Collectively, these results indicate that the presence of anti-LT antibodies may alter the immune modulatory properties of LT promoting a Th1-biased response profile in comparison with the more balanced Th1/Th2 response pattern usually seen in mice co-administered with LT and soluble antigens.

\section{Discussion}

Modern subunit vaccines require potent and versatile adjuvants for inducing efficient and long live protective immunity. Heat-labile toxins derived from ETEC have strong adjuvant effects, demonstrated both at preclinical and clinical conditions, but suffer with safety concerns according to the administration route. In addition, LT derivatives show inherent immunogenicity that may preclude the efficacy of LT-adjuvanted vaccines at endemic regions or at conditions of multiple immunizations. Although this issue has been previously investigated following mucosal delivery, the impact of preexisting immunity on the adjuvant properties of LT in vaccine formulations delivered via parenteral routes remains to be elucidated. In this study, we investigated whether preexisting immunity impairs the humoral and cellular adjuvant properties of LT following s.c. vaccination and our findings demonstrated that previous immunity did not impact the humoral adjuvant activity of LT. We observed also that preexisting immunity did not reduce the local inflammatory signs induced by native LT but our results demonstrated that the presence of LT$\mathrm{Ab}$ immune complexes shift the cytokine expression patterns of both innate and adaptive immune cells. Collectively, the present study opens perspectives for the use of LT, and its derivatives, as parenteral adjuvants and brings relevant information regarding immune response modulation by this class of adjuvants.

In prior studies, preexisting immunity against CT or LT did not impair the antigen-specific antibodies responses induced by this class of adjuvants following mucosal administration[21-23]. Nonetheless, i.n. delivered LT administration, including non-toxic derivatives, has raised safety issues for humans[3,30,31], while similar concerns have not been observed following non-toxic LT administration by parenteral routes. Preclinical studies based on LT derivatives delivered via parenteral routes have demonstrated that these molecules are safe and robust inducers of humoral and cellular immune responses[8-11]. In this context the present study is the first to demonstrate that preexisting immunity to LT did not affect immunogenicity and humoral adjuvant effects of LT, thus adding another supporting evidence for the potential usefulness of LT as parenteral delivered adjuvants.

Here we have also seen that exposure of LT to anti-toxin antibodies change the cytokine secretion pattern of immune cells biasing antigen-specific $\mathrm{T}$ cell responses to the Th1 type. 
In concordance with this result, we detected increased IL- 6 and IL-12 levels at the inoculation site in mice treated with LT-Ab complexes. In fact, IL-12 is strongly associated to Th1 polarization and to immunosuppression of Th2-type response[32-34]. On the other hand, IL- 6 generally leads to $B$ cell activation and a Th2 phenotype[35-37]. However, previous reports have demonstrated that the IL- 6 pleiotropic effects may either lead naive Th cells to differentiate into Th1 or yet not interfere in the $\mathrm{T}$ helper cell profile when IL-12 is up regulated[38-40]. Based on our findings, previous immunity against LT may change proinflammatory cytokines produced at the inoculation site, and, thereby, fine-tune the pattern of the adaptive immune responses mediated by T lymphocytes.

Previous study has demonstrated that neutrophils represent the predominant immune cells infiltrated into edema observed after i.d. inoculation of native LT[8]. Here, we demonstrated that LT is capable of generating edema and neutrophil recruitment to the inoculation site, concomitantly with local IL-1 $\beta$ increases and systemic activation of Th17-type response. In addition, our results demonstrated that the presence of preexisting immunity to LT did not reduce these parameters of inflammatory reactions. Our findings also show that the LT-soluble GM1 complexes drastically reduce swelling diameter and neutrophil migration, as well as strongly decrease the IL-1 $\beta$ and IL-17 levels. Consistently with our findings, IL-1 $\beta$ has been implicated with neutrophil recruitment or, together with IL1- $\alpha$ and IL-23, on modulation of CD4+ T cell to the Th17 profile[41-43]. Moreover, data in the literature demonstrated that LT enhances antigen-specific Th17 cells in an IL-1 $\beta$ and inflammasome activation dependent manner[42]. Thus, the present evidences support the involvement of IL-1 $\beta$ on the modulation of Th17 cells and local neutrophil recruitment, but further studies are required to elucidate the additional role of other proinflammatory cytokines and chemokines.

In conclusion, the evidences presented here demonstrate that preexisting immunity does not negatively impact the humoral adjuvant effects of LT following s.c. vaccination, but may alter the cytokine signatures of innate immune cells and the antigenspecific adaptive immunity biasing it to a Th1 pattern. Our findings, together with those published previously by our research group and others, demonstrate that parenteral administration routes represent robust, safe and efficacious alternatives to delivery of LT-based adjuvants for the mounting of effector humoral and cellular immunity despite preexisting immunity to the toxin.

\section{Acknowledgments}

This study was supported by grants from Fundação de Amparo à Pesquisa do Estado de São Paulo [FAPESP grant number: 2014/17595-0], Conselho Nacional de Desenvolvimento Científico e Tecnológico [CNPq grant number: 485546/20124] and Coordenação de Aperfeiçoamento de Pessoal de Nível Superior [CAPES]. We thank the helpful technical assistance of E. G. Martins and C. Bertelli. All authors confirm that there is no conflict of interest.

\section{References}

1. Nataro JP, Kaper JB. Diarrheagenic Escherichia coli. Clin Microbiol Rev
1998;11(1):142-201.

2. Gomes TAT, Elias WP, Scaletsky ICA, Beatriz EC, et al. Diarrheagenic Escherichia coli. Brazilian Journal of Microbiology. 2016; 47(1):3-30.

3. Giudice GD, Rappuoli R. Genetically derived toxoids for use as vaccines and adjuvants. Vaccine. 1999;17(2):44-52.

4. Norton EB, Lawson LB, Freytag LC, Clements JD. Characterization of a Mutant Escherichia coli heat-labile toxin, LT(R192G/L211A), as a safe and effective oral adjuvant. Clin Vaccine Immunol. 2011;18(4):546551.

5. Rodrigues JF, Mathias-Santos C, Sbrogio-Almeida ME, Amorim JH, Cabrera-Crespo J, Balan A, et al. Functional diversity of heat-labile toxins (LT) produced by enterotoxigenic Escherichia coli. J Biol Chem. 2011;286(7):5222-5233.

6. Holmgren J, Bourgeois L, Carlin N, Clements J, Gustafsson B, Lundgren $A$, et al. Development and preclinical evaluation of safety and immunogenicity of an oral ETEC vaccine containing inactivated E. coli bacteria over expressing colonization factors CFA/I, CS3, CS5 and CS6 combined with a hybrid LT/CT B subunit antigen, administered alone and together with dmLT adjuvant. Vaccine. 2013;31(20):2457-2464.

7. Giuliani MM, Giudice G Del, Giannelli V, Dougan G, Douce G, Rappuoli $\mathrm{R}$, et al. Mucosal adjuvanticity and immunogenicity of LTR72, a novel mutant of Escherichia coli heat-labile enterotoxin with partial knockout of ADP-ribosyltransferase activity. J Exp Med. 1998;187(7):11231132.

8. Hu JC, Mathias-Santos C, Greene CJ, King-Lyons ND, Rodrigues JF, Hajishengallis $\mathrm{G}$ et al. Intradermal administration of the type II heatlabile enterotoxins LT-IIb and LT-IIc of enterotoxigenic Escherichia coli enhances humoral and CD8+ T cell immunity to a co-administered antigen. PLoS One. 2014; 9(12):113978.

9. Armstrong ME, Lavelle EC, Loscher CE, Lynch MA, Mills KHG. Proinflammatory responses in the murine brain after intranasal delivery of cholera toxin: implications for the use of $A B$ toxins as adjuvants in intranasal vaccines. J Infect Dis. 2005; 192(9):1628-1633.

10.Zoeteweij JP, Epperson DE, Porter JD, Zhang CX, Frolova OY, Constantinides AP, et al. GM1 binding-deficient exotoxin is a potent noninflammatory broad spectrum intradermal immunoadjuvant. J Immunol. 2006;177(2):1197-1207.

11. Braga CJM, Rodrigues JF, Medina-Armenteros Y, Farinha-Arcieri LE, Ventura AM, Boscardin SB, et al. Parenteral adjuvant effects of an enterotoxigenic Escherichia colinatural heat-labile toxin variant. Front Immunol. 2014;4:1-11.

12. Petrovsky N, Aguilar JC. Vaccine adjuvants: current state and future trends. Immunol Cell Biol. 2004;82(5):488-496.

13. Saxena M, Van TTH, Baird FJ, Coloe PJ, Smooker PM. Pre-existing immunity against vaccine vectors - friend or foe? Microbiology. 2013;159(1):1-11.

14. Konini A, Kang M, Moghadas SM. Simulating immune interference on the effect of a bivalent glycoconjugate vaccine against Haemophilus influenzae serotypes "a" and "b." Canadian Journal of Infectious Diseases and Medical Microbiology. 2016;1-8.

15. Levine MM, Nalin DR, Hoover DL, Bergquist EJ, Hornick RB, Young CR. Immunity to enterotoxigenic Escherichia coli. Infect Immun. 1979;23(3):729-736.

16. Flores J, DuPont HL, Jiang Z, Belkind-Gerson J, Mohamed JA, Carlin LG, et al. Enterotoxigenic Escherichia coli heat-labile toxin seroconversion in US travelers to Mexico. J Travel Med. 2008;15(3):156-161. 
17. Kauffman RC, Bhuiyan TR, Nakajima R, et al. Single-cell analysis of the plasmablast response to Vibrio cholera demonstrates expansion of cross-reactive memory B cells. MBio. 2016;7(6).

18. Chakraborty S, Harro C, DeNearing B, Ram M, Feller A, Cage A, et al. Characterization of mucosal immune responses to enterotoxigenic Escherichia coli vaccine antigens in a human challenge model: response profiles after primary infection and homologous rechallenge with strain H10407. Clin Vaccine Immunol. 2016;23(1):55-64.

19. Stoll BJ, Svennerholm AM, Gothefors L, Barua D, Huda S, Holmgren J. Local and systemic antibody responses to naturally acquired enterotoxigenic Escherichia coli diarrhea in an endemic area. J Infect Dis. 1986;153(3):527-534.

20. Akhtar M, Qadri F, Bhuiyan TR, Akter S, Rafique TA, Khan A, et al Kinetics of antibody-secreting cell and fecal IgA responses after oral cholera vaccination in different age groups in a cholera endemic country. Vaccine. 2017;35(2):321-328.

21. Tamura S, Funato H, Nagamine T, Aizawa C, Kurata T. Effectiveness of cholera toxin B subunit as an adjuvant for nasal influenza vaccination despite pre-existing immunity to CTB. Vaccine. 1989;7(6):503-505.

22. Lycke N, Schön K. The B cell targeted adjuvant, CTA1-DD, exhibits potent mucosal immunoenhancing activity despite pre-existing antitoxin immunity. Vaccine. 2001;19(17-19):2542-2548.

23. Ugozzoli M, Santos G, Donnelly J, O'Hagan DT. Potency of a genetically detoxified mucosal adjuvant derived from the heat-labile enterotoxin of Escherichia coli (LTK63) is not adversely affected by the presence of preexisting immunity to the adjuvant. J Infect Dis. 2001;183(2):351354.

24. Amorim JH, Porchia BFMM, Balan A, Cavalcante RC, da Costa SM, de Barcelos Alves AM, et al. Refolded dengue virus type 2 NS1 protein expressed in Escherichia coli preserves structural and immunological properties of the native protein. J Virol Methods. 2010;167(2):186192.

25. Lasaro MA, Mathias-Santos C, Rodrigues JF, Ferreira LCS. Functional and immunological characterization of a natural polymorphic variant of a heat-labile toxin (LT-I) produced by enterotoxigenic Escherichia coli (ETEC). FEMS Immunol Med Microbiol. 2009;55(1):93-99.

26. Mathias-Santos C, Rodrigues JF, Sbrogio-Almeida ME, Connell TD, Ferreira LCS. Distinctive immunomodulatory and inflammatory properties of the Escherichia coli type II heat-labile enterotoxin LT-IIa and its B pentamer following intradermal administration. Clin Vaccine Immunol. 2011;18(8):1243-1251.

27. Fraser SA, Haan L de, Hearn AR, Bone HK, Salmond RJ, Rivett AJ, et al. Mutant Escherichia coli heat-labile toxin B subunit that separates toxoid-mediated signaling and immunomodulatory action from trafficking and delivery functions. Infect Immun. 2003;71(3):15271537.

28. Bradley PP, Priebat DA, Christensen RD, Rothstein G. Measurement of cutaneous inflammation: estimation of neutrophil content with an enzyme marker. J Invest Dermatol. 1982;78(3):206-209.

29. Wiig H, Aukland $\mathrm{K}$, Tenstad O. Isolation of interstitial fluid from rat mammary tumors by a centrifugation method. Am J Physiol - Hear Circ Physiol. 2003;284(1):416-424.
30. Mutsch M, Zhou W, Rhodes P, Bopp M, Chen RT, Linder T, et al. Use of the inactivated intranasal influenza vaccine and the risk of Bell's palsy in Switzerland. N Engl J Med. 2004;350(9):896-903.

31. Lewis DJM, Huo Z, Barnett S, Kromann I, Giemza R, Galiza E, et al. Transient facial nerve paralysis (Bell's Palsy) following intranasal delivery of a genetically detoxified mutant of Escherichia coli heat labile toxin. PLoS One. 2009;4(9):6999.

32. Tang C, Rolland JM, Ward C, Li X, Bish R, Thien F, et al. Modulatory effects of alveolar macrophages on CD4+ T-cell IL-5 responses correlate with IL-1beta, IL-6, and IL-12 production. Eur Respir J. 1999;14(1):106-112.

33. Biedermann T, Röcken M, Carballido JM. Th1 and Th2 lymphocyte development and regulation of Thcell-mediated immune responses of the skin. J Investig Dermatology Symp Proc. 2004;9(1):5-14.

34. Jones LL, Vignali DAA. Molecular interactions within the IL-6/IL-12 cytokine/receptor superfamily. Immunol Res. 2011; 51(1):5-14.

35. Rincón M, Anguita J, Nakamura T, Fikrig E, Flavell RA. Interleukin (IL)6 directs the differentiation of IL-4-producing CD4+ T cells. J Exp Med. 1997;185(3):461-469.

36. Tsukamoto H, Senju S, Matsumura K, Swain SL, Nishimura Y. IL-6mediated environmental conditioning of defective Th1 differentiation dampens antitumour immune responses in old age. Nat Commun. 2015;6:6702.

37. Rath T, Billmeier U, Waldner MJ, Atreya R, Neurath MF. From physiology to disease and targeted therapy: interleukin- 6 in inflammation and inflammation-associated carcinogenesis. Arch Toxicol. 2015;89(4):541-554.

38. Yamamoto M, Yoshizaki K, Kishimoto T, Ito H. IL-6 is required for the development of Th1 cell-mediated murine colitis. J Immunol. 2000;164(9):4878-4882.

39. Mayer A, Debuisson D, Denanglaire S, Eddahri F, Fievez L, Hercor $M$, et al. Antigen presenting cell-derived IL-6 restricts Th2-cell differentiation. Eur J Immunol. 2014;44(11):3252-3262.

40. Kumar P, John V, Marathe S, Das G, Bhaskar S. Mycobacterium indicus pranii induces dendritic cell activation, survival, and Th1/Th17 polarization potential in a TLR-dependent manner. J Leukoc Biol. 2015;97(3):511-520.

41. Miller LS, Pietras EM, Uricchio LH, Hirano K, Rao S, Lin H, et al. Inflammasome-mediated production of il-1 is required for neutrophil recruitment against Staphylococcus aureus in vivo. J Immunol. 2007;179(10):6933-6942.

42. Brereton CF, Sutton CE, Ross PJ, Iwakura Y, Pizza M, Rappuoli R, et al. Escherichia coliheat-labile enterotoxin promotes protective Th17 responses against infection by driving innate IL-1 and IL-23 production. J Immunol. 2011;186(10):5896-5906.

43. Chen KW, Groß CJ, Sotomayor FV, Stacey KJ, Tschopp J, Sweet MJ, et al. The neutrophil NLRC4 inflammasome selectively promotes IL$1 \beta$ maturation without pyroptosis during acute Salmonella challenge. Cell Rep. 2014;8(2):570-582. 

3(1): 1-9. 